Trauma Surgery \& Acute Care Open

\title{
Routine repeat head CT may not be necessary for patients with mild TBI
}

\author{
Claire B Rosen, ${ }^{1}$ Diego D Luy, ${ }^{2}$ Molly R Deane, ${ }^{3}$ Thomas M Scalea, ${ }^{1}$ Deborah M Stein ${ }^{1}$
}

'Department of Surgery, University of Maryland School of Medicine, University of Maryland Medical Center, R Adams Cowley Shock Trauma Center, Baltimore, Maryland, USA

2Johns Hopkins School of Medicine, Baltimore, Maryland, USA

${ }^{3}$ Los Angeles County HarborUCLA Medical Center, Department of Surgery, Torrance, California, USA

\section{Correspondence to} Dr Thomas M Scalea, Department of Surgery, University of Maryland School of Medicine, University of Maryland Medical Center, $R$ Adams Cowley Shock Trauma Center, Baltimore, MD 21201 , USA; tscalea@umm.edu

Received 16 August 2017 Revised 14 November 2017 Accepted 13 December 2017

\section{ABSTRACT}

Background Routine repeat cranial CT (RHCT) is standard of care for CT-verified traumatic brain injury (TBI). Despite mixed evidence, those with mild TBI are subject to radiation and expense from serial CT scans. Thus, we investigated the necessity and utility of RHCT for patients with mild TBI. We hypothesized that repeat head CT in these patients would not alter patient care or outcomes.

Methods We retrospectively studied patients suffering from mild TBI (Glasgow Coma Scale (GCS) score 13-15) and treated at the R Adams Cowley Shock Trauma Center from November 2014 through January 2015. The primary outcome was the need for surgical intervention. Outcomes were compared using paired Student's t-test, and stratified by injury on initial CT, GCS change, demographics, and presenting vital signs (mean \pm SD). Results Eighty-five patients met inclusion criteria with an average initial GCS score $=14.6 \pm 0.57$. Our center sees about 2800 patients with TBI per year, or about 230 per month. This includes patients with concussions. This sample represents about $30 \%$ of patients with TBI seen during the study period. Ten patients required operation (four based on initial CT and others for worsening GCS, headaches, large unresolving injury). There was progression of injury on repeat CT scan in only two patients that required operation, and this accompanied clinical deterioration. The mean brain Abbreviated Injury Scale (AIS) score was $4.8 \pm 0.3$ for surgical patients on initial CT scan compared with $3.4 \pm 0.6$ $(\mathrm{P}<0.001)$ for non-surgical patients. Initial $C T$ subdural hematoma size was $1.1 \pm 0.6 \mathrm{~cm}$ for surgical patients compared with $0.49 \pm 0.3 \mathrm{~cm}(P=0.05)$ for non-surgical patients. There was no significant difference between intervention groups in terms of other intracranial injuries, demographics, vital signs, or change in GCS. Overall, 75 patients that did not require surgical intervention received RHCT. At $\$ 340$ per $C T$, $\$ 51000$ was spent on unnecessary imaging (\$367 000/year, extrapolated). Discussion In an environment of increased scrutiny on healthcare expenditures, it is necessary to question dogma and eliminate unnecessary cost. Our data questions the use of routine repeat head CT scans in every patient with anatomic TBI and suggests that clinically stable patients with small injury can simply be followed clinically.

Level of evidence Level III.

\section{INTRODUCTION}

To cite: Rosen CB, Luy DD, Deane MR, et al. Trauma Surg Acute Care Open 2018;3:1-5.
Traumatic brain injury (TBI) affects 1.7 million people each year in the USA. ${ }^{1}$ Although patients at risk for TBI are identified clinically at the time of initial presentation, the specific diagnosis is virtually always made with a head CT scan. ${ }^{2}$ Patients with TBI and a negative CT are usually observed clinically, though some who pose special risks, such as those who are on systemic anticoagulation, ${ }^{3}$ may have a CT scan repeated early in their course.

In virtually every institution, those with an anatomic brain injury diagnosed at admission CT are admitted and observed. In many institutions, a head CT scan is repeated. ${ }^{45}$ This is based on the belief that progression of injury usually occurs by 24 hours, most commonly within 6 hours, and may be difficult to detect clinically. ${ }^{6}$ Thus, repeat CT at 6 and 24 hours is part of many institution's protocols. Although this may be the safest course in patients who are obtunded and/or intubated, the value of repeat CT in patients who are awake and able to be evaluated clinically is less clear.

Repeat CT scanning is not without complications. CT exposes patients to potentially damaging radiation and can increase the cost of healthcare. However, missing the progression of a brain injury can cause substantial morbidity and even mortality. ${ }^{4}$ Mild TBI is often defined as patients who are awake and have a Glasgow Coma Scale (GCS) score of $13-15 .{ }^{8}$ Repeat CT may not be necessary in this particular group of patients, ${ }^{9-11}$ and currently there is inconclusive evidence on how to approach these patients. $^{12}$

We retrospectively reviewed the trauma registry at a major academic trauma center to determine whether repeat head CT scanning in patients with anatomic brain injury seen at admission CT and a GCS score of 13-15 changed care. We hypothesized that repeat head CT in these patients would not alter patient care or outcomes. Secondary aims of this study were to identify what factors, such as injury pattern on diagnostic CT or clinical progression, predict the need for surgical intervention.

\section{METHODS}

We included patients who were treated for mild TBI, defined by GCS score 13-15, between December 2014 and January 2015 at the R Adams Cowley Shock Trauma Center that were identified through the Shock Trauma Registry. A chart review was performed to extract demographic information and patient data pertinent to TBI and treatment. Patients were excluded if found to have a GCS score $<13$ between time of injury and original diagnostic CT, if there was no injury present on initial CT scan, or if initial CT report was unable to be located for analysis.

During the study period, per protocol, patients with suspected TBI had a CT scan. If the admission CT had an injury, patients had a neurosurgical 


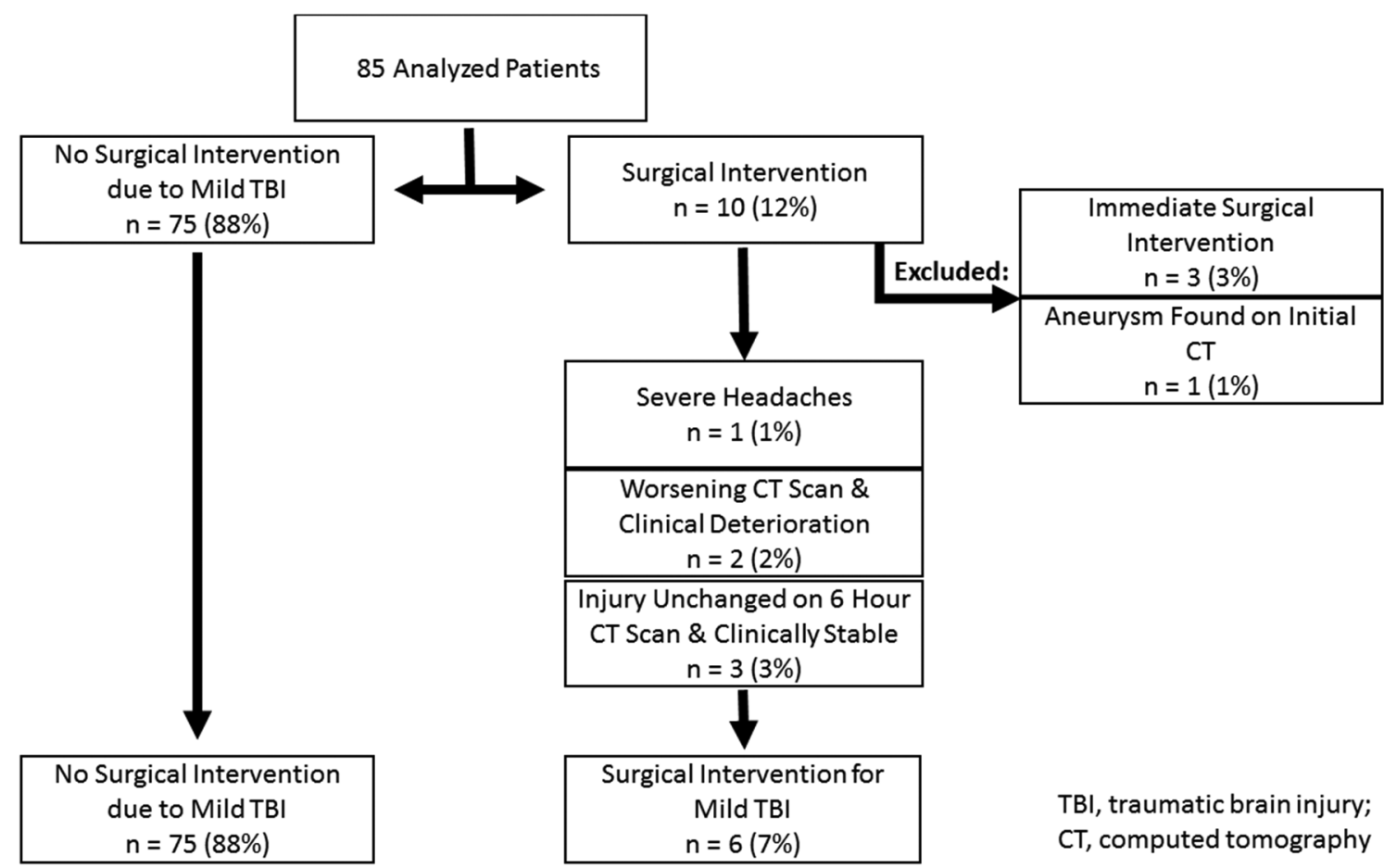

Figure 1 Intervention groups.

consultation, hourly neurological evaluations (including GCS) by nursing staff, antiseizure prophylaxis as indicated, and repeat head CT scans per institutional protocol at 6 and 24 hours (RHCT protocol). There were some patients who did not receive full RHCT protocol as it was deemed unnecessary after neurosurgical consult (due to minimal injury and lack of clinical symptoms) or because they underwent neurosurgical intervention prior to repeat scans. These patients were included in analysis of the 0-hour and 6-hour scans when performed.

Outcomes were compared for patients receiving surgical intervention (including placement of extraventricular or intraventricular drain, craniectomy, craniotomy, or burr-hole washout) versus those who had no intervention, stratified by injury on initial CT (Abbreviated Injury Scale (AIS) and size of subdural hematoma (SDH)), change in GCS, demographics, and presenting vital signs (mean $\pm \mathrm{SD}$ ).

Categorical data were compared using t-tests for two samples assuming unequal variances and reported as significant based on difference between statistic $t$ value and critical $t$ value for a two-tailed analysis. Statistical significance was set at $\mathrm{P} \leq 0.05$.

\section{RESULTS}

Eighty-five patients met inclusion criteria. Our center sees about 2800 patients with TBI per year, or about 230 per month. This includes patients with concussions. This sample represents about $30 \%$ of patients with BTI seen during the study period. Ten patients required surgical intervention: three due to findings on initial CT (including one aneurysm), and the rest for worsening GCS, severe headaches, and large not progressing injury on CT scan (figure 1). In total, $71 \%$ of analyzed patients were male, and they were $74 \%$ Caucasian (table 1). Patients were, on average, overweight with a mean body mass index (BMI) of $27 \pm 6 \mathrm{~kg} /$ $\mathrm{m}^{2}$. The majority of injury were blunt trauma (96\%) and $58 \%$ due to falls. Of note, there were no recorded deaths due to mild TBI among the study population. The mean admission GCS score was $14.6 \pm 0.6$ for all patients, no matter the intervention outcome.

There were no significant differences between outcome groups in terms of demographics or presenting hemodynamics and vital signs: patients in both groups were mostly male, white, and $>60$ years of age (table 2). At admission, patients were hypertensive, with a mean systolic blood pressure of $149 \pm 30 \mathrm{~mm} \mathrm{Hg}$ for those requiring no surgical intervention and $150 \pm 33 \mathrm{~mm} \mathrm{Hg}$ for those requiring surgical intervention $(\mathrm{P}=0.46)$.

Other presenting vital signs were within normal limits and did not statistically differ between intervention groups: peripheral pulse was $83 \pm 19$ beats per minute (BPM) for those not requiring

\begin{tabular}{ll}
\hline Table $1 \quad$ Population demographics \\
\hline Patients, $\mathrm{n}$ & 85 \\
\hline Age, mean $\pm \mathrm{SD}$ & $64 \pm 20$ \\
\hline Gender M (\%)/F(\%) & $60(71 \%) / 25(29 \%)$ \\
\hline Race W $(\%) / \mathrm{B}(\%) / 0(\%)$ & $63(74 \%) / 17(20 \%) / 5(6 \%)$ \\
\hline BMI, mean $\pm \mathrm{SD}\left(\mathrm{kg} / \mathrm{m}^{2}\right)$ & $27 \pm 6$ \\
\hline Mechanism of injury & $18(21 \%)$ \\
\hline Motor vehicle collision, $\mathrm{n}(\%)$ & $1(1 \%)$ \\
\hline Gunshot wound, $\mathrm{n}(\%)$ & $49(58 \%)$ \\
\hline Fall, $\mathrm{n}(\%)$ & $17(20 \%)$ \\
\hline Other, $\mathrm{n}(\%)$ & \\
\hline Mechanism of trauma & $82(96 \%)$ \\
\hline Blunt, $\mathrm{n}(\%)$ & $3(4 \%)$ \\
\hline Penetrating, $\mathrm{n}(\%)$ & $14.6 \pm 0.57$ \\
\hline GCS at admission & $6.3 \pm 8.1$ \\
\hline LOS, mean $\pm \mathrm{SD}$ (days) & $0(0 \%)$ \\
\hline Mortality, $\mathrm{n}(\%)$ & \\
\hline
\end{tabular}

B, black; BMI, body mass index; F, female; GCS, Glasgow Coma Scale; LOS, length of stay; $M$, male; 0 , others; $W$, white. 
Table 2 Demographics by intervention

\begin{tabular}{llll}
\hline & No intervention & Surgical intervention & P value \\
\hline Age, mean \pm SD & $62.2 \pm 20.7$ & $67.5 \pm 16.9$ & 0.27 \\
Male, $\mathrm{n}(\%)$ & $55(73 \%)$ & $4(67 \%)$ & 0.37 \\
\hline $\begin{array}{l}\text { Female, } \mathrm{n}(\%) \\
\text { Black, } \mathrm{n}(\%)\end{array}$ & $20(27 \%)$ & $2(33 \%)$ & \\
$\begin{array}{l}\text { White, } \mathrm{n}(\%) \\
\text { Other, } \mathrm{n}(\%)\end{array}$ & $55(73 \%)$ & $2(33 \%)$ & \\
$\begin{array}{l}\text { Body mass index, } \\
\text { mean } \pm \text { SD }\end{array}$ & $6(8 \%)$ & $0(0 \%)$ & 0.38 \\
\hline
\end{tabular}

surgical intervention versus $75 \pm 9.6 \mathrm{BPM}(\mathrm{P}=0.07)$ for those requiring operation. The respiratory rate was $19 \pm 4.9$ breaths per minute versus $22 \pm 8.1(\mathrm{P}=0.20)$, oxygen saturation was $98 \pm 2.6$ percent oxygen versus $98 \pm 1.3(\mathrm{P}=0.11)$, and diastolic blood pressure was $83 \pm 19 \mathrm{~mm} \mathrm{Hg}$ versus $78 \pm 14 \mathrm{~mm} \mathrm{Hg}$ $(\mathrm{P}=0.26)$.

In classifying initial injury, all patients who required surgical intervention had a SDH and a mean brain AIS score of $4.8 \pm 0.3$, compared with mean brain AIS score of $3.4 \pm 0.6$ for patients requiring no intervention $(\mathrm{P}<0.001)$ (table 3$)$. For those requiring operation, initial CT $\mathrm{SDH}$ size averaged $1.1 \pm 0.6 \mathrm{~cm}$ compared with $0.49 \pm 0.3 \mathrm{~cm}$ for patients who did not require intervention ( $\mathrm{P}=0.05)$; however, there was no significant difference in midline shift (table 3 ). For patients who required surgical intervention, there was a mean GCS drop of $-1.8 \pm 2.7$ in the first 6 hours and $-2 \pm 3$ in 24 hours compared with an increase of $+0.06 \pm 0.4$ for patients requiring no intervention in the first 6 hours $(\mathrm{P}=0.09)$ and $+0.06 \pm 0.8$ in 24 hours $(\mathrm{P}=0.09)$ (table 4).

Seventy-five patients received RHCT but underwent no intervention. At approximately $\$ 340$ per CT, there was an estimated $\$ 51000$ in healthcare cost charged to patients on unnecessary imaging in our study period, or $\$ 367000$ per year, extrapolated.

\section{DISCUSSION}

We retrospectively reviewed a group of patients with mild TBI who had anatomic injury at admission head CT in an attempt to define the utility of routine repeat head CT in these patients. In addition, we sought to determine whether there was a specific group of patients who could benefit from repeat CT. We found that the demographics and clinical presentation of patients that did not require surgical intervention were not different from those who needed some form of surgical therapy early in their course. Thus, clinical information at the time of admission does not seem to define the at risk group. In addition, there was no statistically significant difference in the change in GCS between the two groups. However, the two patients who required urgent

\begin{tabular}{clll}
\hline Table 3 & Initial injury classification by intervention & \\
\hline & $\begin{array}{l}\text { No } \\
\text { intervention }\end{array}$ & $\begin{array}{l}\text { Surgical } \\
\text { intervention }\end{array}$ & P value \\
\hline AIS brain & $3.4 \pm 0.6$ & $4.8 \pm 0.3$ & $<0.001$ \\
\hline Patients, $\mathrm{n}$ & 75 & 6 & \\
\hline SDH size (cm) & $0.49 \pm 0.3$ & $1.1 \pm 0.6$ & 0.05 \\
\hline Patients with SDH, $\mathrm{n}$ & 29 & 5 & \\
Midline shift (cm) & $0.49 \pm 0.5$ & $0.56 \pm 0.4$ & 0.38 \\
\hline Patients with midline shift, $\mathrm{n}$ & 6 & 5 & \\
\hline
\end{tabular}

AIS, Abbreviated Injury Scale; SDH, subdural hematoma.
Table 4 Initial Glasgow Coma Scale (GCS) change by intervention group

\begin{tabular}{|c|c|c|c|}
\hline & Initial GCS & Hour 6 GCS & Hour 24 GCS \\
\hline No intervention (75 patients) & 14.6 & 14.6 & 14.6 \\
\hline \multirow{2}{*}{ Surgical intervention (6 patients) } & 15 & 13.1 & 13 \\
\hline & $\Delta 0-6$ hours & $\Delta 6-24$ hours & $\Delta 0-24$ hours \\
\hline No intervention (75 patients) & $0.06 \pm 0.47$ & $-0.01 \pm 0.82$ & $0.06 \pm 0.86$ \\
\hline Surgical intervention (6 patients) & $-1.8 \pm 2.7$ & $-0.12 \pm 2$ & $-2 \pm 3$ \\
\hline$P$ value & 0.09 & 0.44 & 0.09 \\
\hline
\end{tabular}

therapy for worsening CT scans did have a drop in GCS score to $<13$. In fact, this may be valuable information that could be further investigated.

A number of patients underwent immediate surgical therapy based on the clinical presentation and the appearance of their head CT scan. Some of these patients had an initial GCS score of 15. As they were treated with primary surgical therapy, we did not consider them to have mild TBI despite the fact that they were awake. Thus, we excluded them from our analysis. In addition, three patients had stable GCS and a stable CT scan at 6 hours but were still treated surgically. Two of those patients had their initial CT scan done at an outside institution and were transferred to us. It seems likely that they would have had primary surgical therapy had they been admitted directly to us from the scene. Only two patients had worsening head CT scans. Only two patients failed observation. Thus, the vast majority of patients were successfully observed.

Head CT scan is the diagnostic test used in virtually every trauma center to define the anatomy of the injured brain in patients with TBI. ${ }^{2}$ Patients with severe injury, often characterized by large hematomas and/or signs of elevated intracranial pressure, often have primary surgical treatment. ${ }^{13}$ All others are observed. $^{214}$ Observation usually involves serial neurological examinations. In patients who are multiply injured, intubated, and/or have marked decreases in level of consciousness, serial neurological examinations may be difficult or impossible. In those patients, routine repeat head CT is often used to gauge progression of disease anatomy.

Traditional thinking is that approximately $15 \%$ to $30 \%$ of patients who have a diagnosed brain injury on initial CT scanning will progress within the first 24 hours. ${ }^{615-17}$ Most commonly, this is thought to happen during the first 6 hours. ${ }^{14}$ Therefore, in many trauma centers, including ours, head CT scans are routinely repeated at 6 and 24 hours in most patients with an anatomic brain injury identified on the initial head CT scan. CT scans may also be repeated in very high-risk patients with a normal head CT, such as those on systemic anticoagulation. ${ }^{3}$

Head CT accurately describes injury anatomy. However, CT is not a good functional assessment of the injured brain. Mild TBI is usually defined as patients who are awake, with a GCS score of $13-15 .{ }^{8}$ Although routine repeat head CT seems rational in patients who cannot be followed clinically, their role is less clear in patients with mild TBI who are awake and can be more accurately evaluated with serial neurological examinations. ${ }^{17}$

The role of repeat head CT has been debated. Currently, there is not conclusive evidence that repeat head CT helps. In fact, several studies have demonstrated that clinical care does not change, regardless of the repeat head CT results without accompanying clinical deterioration. ${ }^{5} 91018$ However, many of these studies have included all patients with TBI and do not focus specifically on patients with mild TBI, which account for $75 \%$ of 
all patients with TBI. ${ }^{8}$ In 2012, AbdelFattah et al looked at the use of RHCT in patients with intracranial hemorrhage and GCS score $13-15$ in a prospective study of 145 patients with TBI. ${ }^{19}$ They found that selective use of RHCT decreased hospital length of stay in these patients, but they did not use injury pattern on initial CT scan to allocate patients to routine or selective repeat scans.

There was a statistically significant difference in brain AIS between those who required surgical intervention and those who did not. The group requiring operation had an average AIS of 4.8, impressively high for a group of patients that were still awake. Thus, it would seem that injury severity at the time of patient presentation is an important factor in predicting who may fail observation and require urgent therapy. If we were to further exclude patients with AIS score $>3$ analysis by identifying them as patients with more than mild TBI, then no patients in our study would have required surgical intervention.

In addition, the size of the SDH seems to be important. Although the $\mathrm{P}$ value for SDH size was not quite statistically significant, it is likely clinically important. One would guess that the relatively small sample size failed to allow us to identify SDH size as significant. Although SDH size was important, the degree of shift was not. This is also a little unexpected and will require further work to determine its level of importance.

In 2014, Phelan et al found that isolated traumatic subarachnoid hemorrhage required significantly fewer head CT scans than other forms of TBI. ${ }^{20}$ Most studies fail to consider the information provided on the head CT scan done at admission. It may be possible to define a group of patients who are at particular risk for progression of injury on repeat CT, perhaps those with large SDH. This would allow clinicians to concentrate efforts on these high-risk patients, eliminating the blanket use of repeat CT.

Classification of injury on diagnostic CT scan guides patient care. For example, the Parkland Protocol is an algorithm that categorizes TBI patterns as low, moderate, or high risk for spontaneous expansion and suggests venous thromboembolism (VTE) prophylaxis for each group. ${ }^{21} 22$ In this algorithm, later modified to be only two tiered including only low and high risk for injury progression/expansion, ${ }^{23}$ injury size on diagnostic CT scan (eg, SDH $\leq 8 \mathrm{~mm}$ ) and evolution of injury on repeat CT scan at 24 hours, defines risk and direct use of VTE prophylaxis. In a prospective study on incidence and timing of radiographic worsening within this protocol, ${ }^{21}$ it was found that more severe injuries on diagnostic CT scan (moderate-risk or high-risk TBI) are more likely to progress.

Perhaps protocol such as these could be further modified and used to predict which patients require repeat CT imaging to evaluate injury progression. Our patients that required surgical intervention had an average SDH size of $1.1 \mathrm{~cm}$, placing them into the Parkland Protocol algorithm high-risk tier for injury progression, despite these patients fitting our definition of mild TBI. Clearly, further investigation, with larger patient populations and greater power, is necessary to elucidate these associations and guide appropriate use of RHCT.

In an environment of increased scrutiny on healthcare expenditures, it is necessary to question dogma and eliminate unnecessary cost. It seems that routine repeat head CT for every patient with injury seen at admission CT is a practice that needs to be re-evaluated. Clearly, the cost of missing injury progression can be devastating. However, repeating a head CT in a patient with a GCS score of 15 and a tiny SDH likely is unnecessary. The charge for a head CT at our institution is approximately $\$ 340$. This does not include the cost of the radiological interpretation. Thus, had the CT scans not been repeated in the 75 patients that did not require intervention, the institution would have saved $\$ 51000$ during a short period of time. This extrapolated to a savings of $\$ 367000$ per year.

Stein et $a l^{24}$ found mixed results when evaluating cost-effectiveness of routine repeat head CT, asserting that repeat CT is more cost-effective for children but that the cost-effectiveness decreases with age. Of course, the risk associated with radiation exposure is greatest for children. In addition, routine repeat head CT is costly. Institutional charges combined with the radiologist fee for interpretation often are many hundreds of dollars. These are healthcare dollars that could be expended in many other ways.

This is clearly a small study and does not have the power to make strong recommendations. In addition, although the first author (CBR) personally reviewed all of the charts, the data are retrospective. The patients were injured in a short period of time and may not reflect all other time periods. Whereas some patients had isolated TBI, others had associated injuries that may have impacted on clinical decision-making. Additionally, there was no study team member radiologist who independently reviewed CT scans. Furthermore, outcome data were not analyzed, including Glasgow Outcome Scale, discharge locations, or complications.

Despite its limitations, our data further question the use of routine repeat head CT scans in every patient with an anatomic TBI identified at the time of admission. It would seem that patients with larger SDHs and those with more severe anatomic TBI may still benefit from repeat imaging. However, the patient that is awake and alert that has a small SDH likely can simply be followed clinically.

Contributors CBR, MRD, TMS and DMS conceived the study. CBR and TMS analyzed the data, CBR, MRD, TMS, DMS and DDL drafted the manuscript. All authors provided critical feedback and contributed to final revisions.

Funding This research was supported in part by the University of Maryland, School of Medicine, Office of Student Research, the Shock Trauma Registry, The R Adams Cowley Shock Trauma Center, and the University of Maryland Medical System.

\section{Competing interests None declared.}

Ethics approval Approval for retrospective data analysis was obtained from the University of Maryland, Baltimore Institutional Review Board.

Provenance and peer review Not commissioned; externally peer reviewed.

Open Access This is an Open Access article distributed in accordance with the Creative Commons Attribution Non Commercial (CC BY-NC 4.0) license, which permits others to distribute, remix, adapt, build upon this work non-commercially, and license their derivative works on different terms, provided the original work is properly cited and the use is non-commercial. See: http://creativecommons.org/ licenses/by-nc/4.0/

(c) Article author(s) (or their employer(s) unless otherwise stated in the text of the article) 2018. All rights reserved. No commercial use is permitted unless otherwise expressly granted.

\section{REFERENCES}

1 Faul M, Xu L, Wald M, Coronado V. Traumatic brain injury in the United States: emergency department visits, hospitalizations and deaths 2002-2006. Atlanta, GA: Centers for Disease Control and Prevention, National Center for Injury Prevention and Control, 2017.

2 Joseph B, Friese RS, Sadoun M, Aziz H, Kulvatunyou N, Pandit V, Wynne J, Tang A, O'Keeffe T, Rhee P. The BIG (brain injury guidelines) project: defining the management of traumatic brain injury by acute care surgeons. J Trauma Acute Care Surg 2014;76:965-6.

3 Wang MC, Linnau KF, Tirschwell DL, Hollingworth W. Utility of repeat head computed tomography after blunt head trauma: a systematic review. J Trauma 2006;61:226-33.

4 Ding J, Yuan F, Guo Y, Chen SW, Gao WW, Wang G, Cao HL, Ju SM, Chen H, Zhang PQ, et al. A prospective clinical study of routine repeat computed tomography (CT) after traumatic brain injury (TBI). Brain Inj 2012;26:1211-6.

5 Brown CV, Weng J, Oh D, Salim A, Kasotakis G, Demetriades D, Velmahos GC, Rhee $P$. Does routine serial computed tomography of the head influence management of traumatic brain injury? A prospective evaluation. J Trauma 2004;57:939-43. 
6 Brown CV, Zada G, Salim A, Inaba K, Kasotakis G, Hadjizacharia P, Demetriades D, Rhee P. Indications for routine repeat head computed tomography (CT) stratified by severity of traumatic brain injury. J Trauma 2007;62:1339-45.

7 Ron E. Cancer risks from medical radiation. Health Phys 2003;85:47-59.

8 Sternbach GL. The Glasgow coma scale. J Emerg Med 2000;19:67-71.

9 Washington CW, Grubb RL. Are routine repeat imaging and intensive care unit admission necessary in mild traumatic brain injury? I Neurosurg 2012;116:549-57.

10 Connon FF, Namdarian B, Ee JLC, Drummond KJ, Miller JA. Do Routinely Repeated Computed Tomography Scans in Traumatic Brain Injury Influence Management? Ann Surg 2011;254:1028-31.

11 Haydel MJ, Preston CA, Mills TJ, Luber S, Blaudeau E, DeBlieux PM. Indications for computed tomography in patients with minor head injury. N Engl I Med 2000;343:100-5.

12 Reljic T, Mahony H, Djulbegovic B, Etchason J, Paxton H, Flores M, Kumar A. Value of repeat head computed tomography after traumatic brain injury: systematic review and meta-analysis. J Neurotrauma 2014;31:78-98.

13 Kolias AG, Adams H, Timofeev I, Czosnyka M, Corteen EA, Pickard JD, Turner C, Gregson BA, Kirkpatrick PJ, Murray GD, et al. Decompressive craniectomy following traumatic brain injury: developing the evidence base. Br I Neurosurg 2016;30:246-50.

14 Stippler M, Liu J, Motiei-Langroudi R, Voronovich Z, Yonas H, Davis RB. Complicated Mild Traumatic Brain Injury and the Need for Imaging Surveillance. World Neurosurg 2017;105:265-9.

15 Lee B, Newberg A. Neuroimaging in traumatic brain imaging. NeuroRx 2005;2:372-83.

16 Chen H, Guo Y, Chen SW, Wang G, Cao HL, Chen J, Gu Y, Tian HL. Progressive epidural hematoma in patients with head trauma: incidence, outcome, and risk factors. Emerg Med Int 2012;2012:1-8.
17 Homnick A, Sifri Z, Yonclas P, Mohr A, Livingston D. The temporal course of intracranial haemorrhage progression: how long is observation necessary? Injury 2012;43:2122-5.

18 Rubino S, Zaman RA, Sturge CR, Fried JG, Desai A, Simmons NE, Lollis SS. Outpatient follow-up of nonoperative cerebral contusion and traumatic subarachnoid hemorrhage: does repeat head CT alter clinical decision-making? J Neurosurg 2014;121:944-9.

19 AbdelFattah KR, Eastman AL, Aldy KN, Wolf SE, Minei JP, Scott WW, Madden CJ Rickert KL, Phelan HA. A prospective evaluation of the use of routine repeat cranial CT scans in patients with intracranial hemorrhage and GCS score of 13 to 15. J Trauma Acute Care Surg 2012;73:685-8.

20 Phelan HA, Richter AA, Scott WW, Pruitt JH, Madden CJ, Rickert KL, Wolf SE. Does isolated traumatic subarachnoid hemorrhage merit a lower intensity level of observation than other traumatic brain injury? I Neurotrauma 2014;31:1733-6.

21 Phelan HA, Eastman AL, Madden CJ, Aldy K, Berne JD, Norwood SH, Scott WW, Bernstein IH, Pruitt J, Butler G, et al. TBI risk stratification at presentation: a prospective study of the incidence and timing of radiographic worsening in the Parkland Protocol. J Trauma Acute Care Surg 2012;73:S122-S127.

22 Phelan HA. Pharmacologic venous thromboembolism prophylaxis after traumatic brain injury: a critical literature review. J Neurotrauma 2012;29:1821-8.

23 Pastorek RA, Cripps MW, Bernstein IH, Scott WW, Madden CJ, Rickert KL, Wolf SE, Phelan HA. The Parkland Protocol's modified Berne-Norwood criteria predict two tiers of risk for traumatic brain injury progression. J Neurotrauma 2014;31:1737-43.

24 Stein SC, Fabbri A, Servadei F. Routine serial computed tomographic scans in mild traumatic brain injury: when are they cost-effective? I Trauma 2008;65:66-72. 